\title{
Computationally assisted screening and design of cell-interactive peptides by a cell- based assay using peptide arrays and a fuzzy neural network algorithm
}

\author{
Chiaki Kaga ${ }^{1}$, Mina Okochi ${ }^{1}$, Yasuyuki Tomita ${ }^{1}$, Ryuji Kato ${ }^{1}$, and Hiroyuki Honda ${ }^{1}{ }^{12}$
}

BioTechniques 44:393-402 (March 2008)

doi $10.2144 / 000112693$

\begin{abstract}
We developed a method of effective peptide screening that combines experiments and computational analysis. The method is based on the concept that screening efficiency can be enhanced from even limited data by use of a model derived from computational analysis that serves as a guide to screening and combining the model with subsequent repeated experiments. Here we focus on celladhesion peptides as a model application of this peptide-screening strategy. Cell-adhesion peptides were screened by use of a cellbased assay of a peptide array. Starting with the screening data obtained from a limited, random 5-mer library (643 sequences), a rule regarding structural characteristics of cell-adhesion peptides was extracted by fuzzy neural network (FNN) analysis. According to this rule, peptides with unfavored residues in certain positions that led to inefficient binding were eliminated from the random sequences. In the restricted, second random library (273 sequences), the yield of cell-adhesion peptides having an adhesion rate more than 1.5-fold to that of the basal array support was significantly high (31\%) compared with the unrestricted random library (20\%). In the restricted third library (50 sequences), the yield of cell-adhesion peptides increased to $84 \%$. We conclude that a repeated cycle of experiments screening limited numbers of peptides can be assisted by the rule-extracting feature of FNN.
\end{abstract}

\section{INTRODUCTION}

Peptides have attracted attention for their ability to regulate a variety of cellular events by interacting with receptors on the cell surface. A large number of biomaterials that conjugate functional peptides have been developed for medicine, drug delivery, tissue engineering, bio-imaging, and food additives. However, identifying functional peptides by exhaustive screening that covers all the possible combinations of 20 amino acids is terribly time consuming.

Computationally assisted peptide screening and design have become invaluable tools in overcoming this inefficiency in peptide screening. One of the most advanced areas in the computational analysis of peptides is MHC ligand prediction (1-4). The peptides that bind to the highly polymorphic molecule MHC were discovered partially by experiment, and all of the data have been accumulating in databases, such as MHCPEP (http://wehih.wehi.edu.au/mhcpep/) and MHCBN (http://www.imtecfh.res. $\mathrm{in} / \mathrm{reghava} / \mathrm{mhcbn} /$ ). Using these data as training data, quantitative modeling studies on peptides binding to the MHC have been constructed to have high predictive accuracies for distinguishing unknown peptide ligands by use of various types of algorithms, including a Bayesian neural network (1), artificial neural network (ANN) (2), weight matrix (3), inheritable bi-objective genetic algorithm (4), and support vector machines $(5,6)$. There are also strategies that predict peptide folding (7) or interactive relationships with target receptors (8), which should offer powerful support for drug screening. However, the predictive efficiency of most of these methods is evaluated with their own datasets. Repeated confirmations of the peptide interaction assays to validate the robustness of predictors are not usually performed. In our previous work, we constructed a new computational model for predicting MHC class II binding peptides. A fuzzy neural network (FNN) (9) and hidden Markov model (10) were demonstrated to increase the predictive accuracy of an acquired prediction model. Also, it should be noted that existing computational work is often applied only to restricted prediction studies. For example, the MHC binding peptides possess a core binding region of approximately 9-mer, and important positions that are known to match the structure of the cavity in the receptor have been evaluated for their affinity. These biological restrictions can make the modeling process simpler and 


\section{Research Reports}

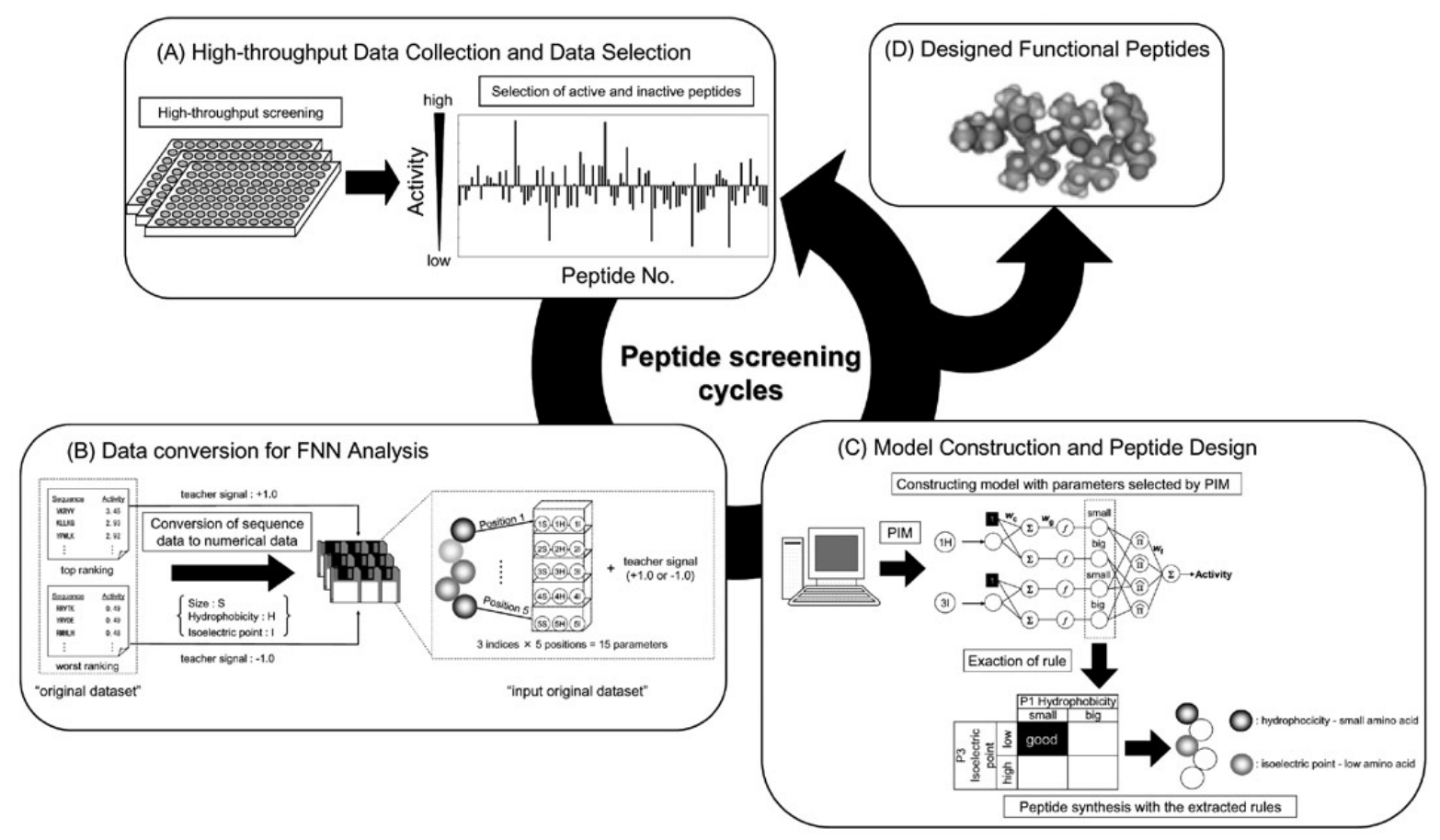

Figure 1. Schematic diagram of fuzzy neural network (FNN)-assisted peptide design. FNN analysis of high-throughput assays enables efficient screening for biological activities using deduced peptide design rules. (A) The high-throughput peptide screening assay using peptide arrays and data collection. The sequences of highly active peptides (top-ranking) and inactive peptides (worst-ranking) from the assayed peptides were used in FNN analysis. (B) Data conversion into input data for FNN analysis. The sequence of each peptide was converted into numerical data by applying indices of size, hydrophobicity, and isoelectric point at each amino acid position. A teacher signal of +1.0 was assigned to high activity peptides and -1.0 to inactive peptides. (C) Construction of the predictive model and peptide design in accordance with extracted rules. The most effective input parameter combination contributing to the peptide sequence-activity relationship was derived by the use of parameter-increasing method (PIM). The constructed predictive model was then used to extract peptide design rules, and peptides were designed in accordance with the extracted peptide design rules for subsequent screening. (D) Peptide design in accordance with optimized rules by repeating peptide screening cycle.

increase predictive accuracy. It would be helpful to develop a new computational tool for general predictive studies having no or only light restrictions.

In this study, we focused on cellpeptide interaction, screening for ideal synthetic peptide sequences to apply to the design of cell-culture scaffolds. Since cells express various receptors on the cell surface, the assay output-that is, the cell-adhesion rate-consists of much complex information. However, by repeated evaluation of the model, we found that such complex interactions can be modeled and that by interpreting the constructed model, structural rules for target peptides can be established as "design rules" through the use of our original tool, the FNN (11).

The FNN is a type of ANN that automatically constructs complex model structures by learning the hidden relationship between input and output data, thus functioning as a predictor. It has been shown that high predictive accuracies can be obtained in the analysis of various data derived from complex phenomena, such as bioprocess factors and fermentation efficiency (12), protein structure information and protein enantioselectivity (13), expression profiles of microarray data and the diagnosis of disease development (14), and the prediction of peptide binding to MHC class II (9). As compared with ANN, FNN has an advantage: the "fuzzy layer." This allows interpretation of the model structure and extraction of the quantified relationship between input and output values as a rule designated as the "if-then" rule. This feature distinguishes FNN from the usual neural network-based models, since the former provides rules for structural design described by combinations of the physical properties of important residues, whereas the latter function only as a black box. We have used this tool to enhance the efficiency of peptide screening when the peptide interaction rules are ambiguous, such as in cell-peptide interactions, which are the focus of this study.

Our strategy is to use repeated highthroughput assays of cell-peptide interactions and computational modeling to derive effective rules for synthesizing target peptides with higher cell-binding activity. We have used FNN as an informative modeling algorithm that can contribute to the design of peptides tested in the high-throughput assay, a cell-based assay performed on a peptide array (15-17).

Peptide arrays (developed as the SPOT method by Ronald Frank) are highly effective in the exhaustive screening of peptides in biomedical and pharmaceutical research (18). The standard solid-phase peptide synthesis protocol for constructing a peptide array involves the spotting of small volumes of activated amino acid solutions at addressed positions on a support such as a cellulose membrane, and the elongation of peptide sequences by the use of 9-fluorenylmethoxy- 


\section{Research Reports}

Table 1. Peptide Design Rules Extracted by Fuzzy Neural Network Analysis

\begin{tabular}{|c|c|c|c|c|}
\hline Rule & P1 & P2 & P3 & P4 \\
\hline \multirow[t]{2}{*}{ Rule 1} & Size: small & Isoelectric point : high & No rule & Size: small \\
\hline & $\begin{array}{c}\text { A, N, D, C, Q, F, G, I, L, M, } \\
\text { P, S, T, V }\end{array}$ & $\mathrm{R}, \mathrm{K}$ & All amino acids & $\begin{array}{c}\text { A, N, D, C, Q, F, G, I, L, M, } \\
\text { P, S, T, V }\end{array}$ \\
\hline \multirow[t]{2}{*}{ Rule 2} & $\begin{array}{c}\text { Size: small } \\
\text { Isoelectric point: } \text { high }\end{array}$ & Isoelectric point : high & $\begin{array}{l}\text { Size: big } \\
\text { Isoelectric point: high }\end{array}$ & Size: small \\
\hline & A, G, I, L, M, P, V & $\mathrm{R}, \mathrm{K}$ & R, H, K, M, F, W, Y & $\begin{array}{c}\text { A, N, D, C, Q, F, G, I, L, M, } \\
\text { P, S, T, V }\end{array}$ \\
\hline
\end{tabular}

carbony (Fmoc) chemistry. Owing to its convenience in biochemical assays, peptide array technology has been used to investigate interactions between peptides on the array and biological molecules $(19,20)$. Previously, we developed a peptide array-based cell assay system that allows screening for functional peptides that directly influence cell behavior, through interaction with cell surface receptors, and we succeeded in identifying celladhesion peptides from fibronectin sequence (15).

The screening of cell-adhesion peptides has commonly been performed using the amino acid sequences of the cell-adhesion proteins fibronectin (21) and laminin (22), or by using peptide libraries that selectively recognize specific cells such as tumor vasculature (23, 24), vascular endothelium (25), or $\mathrm{CD} 11 \mathrm{c} / \mathrm{CD} 18$ positive cells (26). For regenerative medicine and tissue engineering applications, biomaterials conjugated to cell-adhesion peptides have been developed as artificial scaffolds that mimic natural extracellular matrices (27-29). The development of synthetic cell-adhesion ligands that represent the complexity of regulatory functions in extracellular microenvironments has been pursued. Such materials could overcome the safety issues associated with materials of animal origin, including pathogen transmission. Understanding the function and structure of cell-adhesion ligands is also important for clinical applications, since many biological processes, including angiogenesis, tumor invasion, tumor metastasis, tissue repair, inflammation, and atherosclerosis require cell-adhesion and migration. In addition to some relevant peptides such as RGDS, YIGSR,
DEGA, and EILDV, which are known integrin ligands, some of their sequence analogs and other sequences from other extracellular matrix proteins show celladhesive effects. Most of these analogs display weaker effects than the abovementioned short peptides. However, cells express various subtypes of integrin in various quantities on their surfaces, and they are fully active even without recognized short peptide motifs. Therefore, an understanding of the sequence patterns of cell-adhesion peptides could contribute to the design of scaffolds that enhance cellular activity by supporting adhesion.

Here we report the new strategy, which combines peptide array-based high-throughput screening with computational analysis to improve the efficiency of peptide screening in celladhesion peptide discovery.

\section{MATERIALS AND METHODS}

\section{Preparation of Peptide Arrays}

A cellulose membrane (grade 542; Whatman, Maidstone, UK) was activated by using $\beta$-alanine as the basal spacer. Fmoc-11-aminoundecanoic acid (Watanabe Chemical Inc., Hiroshima, Japan) was used as the additional spacer. Activated Fmoc amino acids were spotted using a peptide auto-spotter (ASP222; Intavis AG, Köln, Germany) in accordance with the manufacturer's instructions with some modifications. Activated amino acids at a concentration of $0.5 \mathrm{M}$ were spotted at intervals of $2.0 \mathrm{~mm}$. The membrane was washed with $\mathrm{N}, \mathrm{N}^{\prime}$-dimethylformamide and the Fmoc groups were deprotected with $20 \%$ piperidine. Additional washes were performed with $\mathrm{N}, \mathrm{N}^{\prime}$-dimethylformamide, followed by washing with methanol. These steps were performed in the same order in every elongation step. The final removal of side-chain protecting groups was performed with a reagent mixture comprised of water: dichloromethane:triisopropylsilane: trifluoroacetic acid at a molar ratio of 2:3:50:50 for $3 \mathrm{~h}$. After removal of the protecting group, the membrane was washed several times with diethyl ether and methanol and was allowed to dry.

\section{Cell Culture}

NIH/3T3 mouse fibroblast cells (American Type Culture Collection, Manassas, VA, USA) were cultured in Dulbecco's Minimal Eagle's Medium (DMEM; Invitrogen, Carlsbad, CA, USA) supplemented with $10 \%$ fetal bovine serum (Invitrogen) and $1 \%$ antibiotic-antimitotic mixture (Invitrogen) at $37^{\circ} \mathrm{C}$, under $5 \% \mathrm{CO}_{2}$.

\section{Cell-based Assay on a Peptide Array}

The synthesized peptide array was washed several times with phosphatebuffered saline (PBS; pH 7.0), and the membrane was allowed to dry under sterile conditions. Each peptide spot was punched out as a peptide disk and inserted in a 96-well plate. The NIH/3T3 cells were added to the peptide disk at a cell density of $7.5 \times$ $10^{3}$ cells/well and incubated for $5 \mathrm{~h}$ at $37^{\circ} \mathrm{C}$ under $5 \% \mathrm{CO}_{2}$. The medium was then carefully removed from the wells, which were washed three times with PBS to remove unattached cells. The 96-well plate was shaken at 1200 rpm for $10 \mathrm{~s}$ on a Fluoroskan Ascent fluorescent plate reader (type 374; Labsystems, Helsinki, Finland). The number of cells adhering to the peptide disk was estimated using calcein acetoxymethyl ester (Invitrogen). The fluorescence intensity was measured by using a fluorescent plate reader after $1 \mathrm{~h}$ of incubation at $37^{\circ} \mathrm{C}$ under $5 \%$ $\mathrm{CO}_{2}$. The average value of triplicate experiments was used to estimate the number of cells adhering to each peptide disk. The relative cell-adhesion rate was calculated as the ratio of the number of viable cells remaining on the peptide disk to the number adhering 


\section{Research Reports}

to a membrane lacking peptides (set to $1.0)$.

\section{Peptide Data Conversion}

For the following analysis, the property and positional information of the residues in the peptide sequences were converted into numerical indices. All the residues were converted into three physical properties: hydrophobicity (30), isoelectric point (31), and size (32). By linking these three properties with the positional information, 5-mer residues were converted into a total of 15 input parameters and matched with the relative cell-adhesion rate. For the 15 parameters representing peptide sequences, the teacher signal was assigned a value of +1.0 for the selected sequences in the topranking in relative cell-adhesion rate, and a value of -1.0 for those from the worst-ranking. The 15 input parameters paired with a teacher signal of +1.0 or -1.0 were treated as one datum, designated as a "dataset."

\section{Dataset Arrangements and FNN Model Construction}

The procedure for constructing the model is presented schematically in Supplementary Figure 1 (see Supplementary Materials, available online at www.BioTechniques.com). First, all datasets were randomly arranged and were equally divided into four datasets (original datasets). One of these four datasets was chosen as "blind dataset A1," which was never used in the model construction and only used in the final evaluation of the model. The remaining datasets, "modeling datasets A1," were newly split into four datasets again and subjected to 4-fold crossvalidation $(\mathrm{CV})$, using three datasets as training data and one dataset for validation. Through this 4 -fold $\mathrm{CV}$, the best combination of input parameters, which provides the best predictive accuracy as an average throughout the CV, was selected by the use of the parameter-increasing method (PIM) (13). Blind dataset A1 was evaluated with this parameter combination, and the model's accuracy (designated as accuracy A1) was obtained.

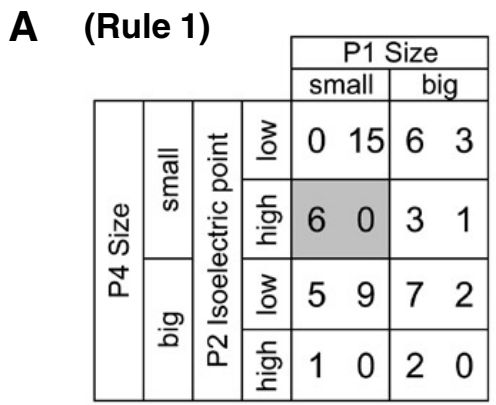

(Active / Inactive)

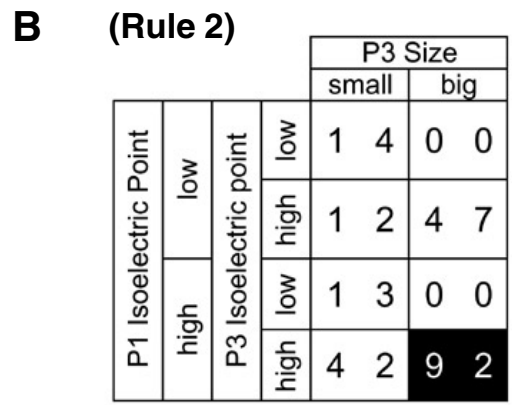

(Active / Inactive)

Figure 2. Rules extracted from the fuzzy neural network (FNN) predictive model for cell-adhesion peptides. (A) Rule 1 and (B) Rule 2 reveal the rule matrix deduced from first and second screening cycles, respectively. In the rule matrix, the numbers of highly active and inactive peptides in the dataset used for constructing the model were counted and placed in the left and right positions, respectively, in each cell. In order to count corresponding peptides in each rule, the 20 amino acids were classified as big or small and their isoelectric points as high or low by the membership functions adjusted in the model structure and used to classify the dataset sequences.

Table 2. Relative Cell-adhesion Ratio of Peptides Designed According to Rule 2

\begin{tabular}{|c|c|c|c|c|c|c|c|}
\hline Peptides & $\begin{array}{r}\text { Relative Cell- } \\
\text { adhesion Ratio }\end{array}$ & $\mathrm{pl}$ & Hydrophobicity & Peptides & $\begin{array}{l}\text { Relative Cell- } \\
\text { adhesion Ratio }\end{array}$ & $\mathrm{pl}$ & Hydrophobicity \\
\hline GKFQ & $3.4 \pm 0.1$ & 10.2 & -5.4 & МKHT & $2.4 \pm 0.2$ & 9.9 & -5.9 \\
\hline IRRC & $3.0 \pm 0.3$ & 11.0 & -2.0 & VKKM & $2.2 \pm 0.1$ & 10.6 & -1.7 \\
\hline VKKP & $3.0 \pm 0.2$ & 10.6 & -5.2 & PKYL & $2.2 \pm 0.3$ & 10.1 & -3.0 \\
\hline PRKQ & $2.9 \pm 0.3$ & 11.7 & -13.5 & MKKN & $2.2 \pm 0.2$ & 10.6 & -9.4 \\
\hline GKKA & $2.9 \pm 0.3$ & 10.6 & -6.4 & ARYD & $2.2 \pm 0.1$ & 6.8 & -7.5 \\
\hline PKHQ & $2.8 \pm 0.3$ & 10.6 & -12.2 & VKRG & $2.2 \pm 0.1$ & 11.5 & -7.7 \\
\hline GKMN & $2.6 \pm 0.2$ & 10.2 & -5.9 & PRFQ & $2.0 \pm 0.3$ & 11.6 & -6.8 \\
\hline VKKV & $2.6 \pm 0.4$ & 10.6 & -0.6 & IRHM & $2.0 \pm 0.1$ & 11.2 & -1.3 \\
\hline PKKN & $2.4 \pm 0.2$ & 10.8 & -12.9 & & & & \\
\hline
\end{tabular}

Next, the process of creating one model with one parameter combination of three inputs was repeated another three times. This process provided blind datasets A2-A4 with accuracy A2-A4 from the original dataset. As a result, four different models with four different combinations of input parameters were obtained.

Next, the above-mentioned first and second steps were repeated three more times, with different data randomization and arrangement. This produced 12 more individual models evaluated by 12 blind datasets: blind datasets B1-B4, C1-C4, and D1-D4. Hence, a final total of 16 individual models with 16 combinations of input parameters were obtained.

The final model for peptide design was then constructed. The first and second parameters were chosen based on the frequency of selection in all 16 models obtained. The third parameter was then selected from the 48 candidate parameters (3 parameters from 16 models) as that which yielded the best predictive accuracy when applied to the first and second parameter in the blind data (16 patterns). In the construction of each model, the learning process was terminated when the model attained the highest predictive accuracy with the test data. The weight adjustment was repeated 1000 times with each step, with the learning coefficient value set to 0.1 . The parameter condition was defined by previous studies $(9,13,14)$.

\section{FNN-assisted Peptide Screening}

From the high-throughput data collection step, 643 randomly selected 5 -mer peptides were chosen from $20^{5}$ possible variants. The highest scoring 30 peptides (teacher signal 1.0) and 


\section{Research Reports}
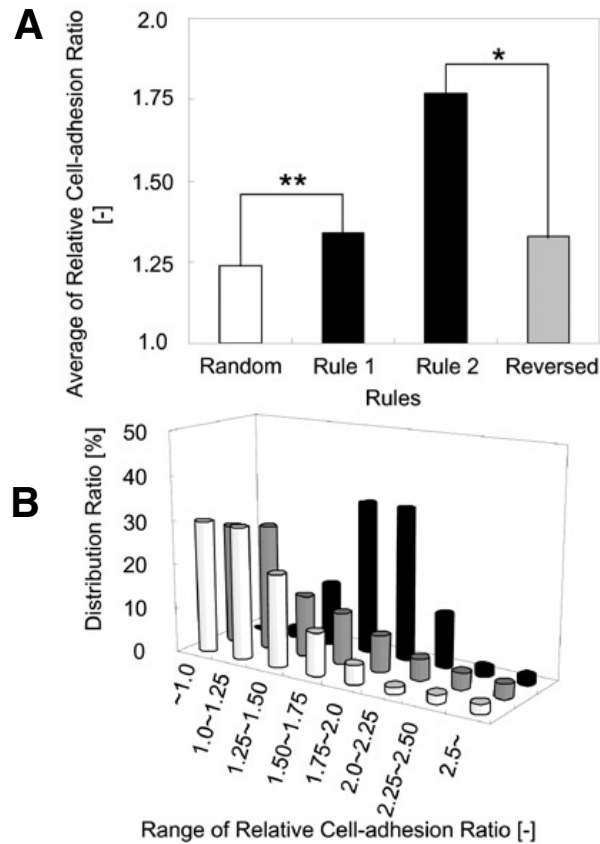

the lowest scoring 30 peptides (teacher signal -1.0) were chosen as the training datasets for the first FNN modeling (Supplementary Table 1). With these datasets designated as the "first original dataset," the best combination of three parameters and the best predictive model using those parameters were obtained through data rearrangement and FNN model construction. From the best FNN model, the rule was obtained by interpreting the weights (Wf) (11) in the model structure. This rule indicated the possible preferred peptide structural tendencies that differentiated celladhesion sequences (teacher signal 1.0) from nonadhesion sequences (teacher signal -1.0). For the next data collection step, peptides were newly synthesized on the array in accordance with this rule. For example, if the rule indicated that a residue of higher hydrophobicity is preferred in the $\mathrm{N}$-terminal position, then sequences with hydrophobic residues (e.g., Val, Ile, Leu) in the $\mathrm{N}$ terminal position were selected to be screened. As a comparative library, randomly selected peptides were prepared in parallel.

In the second cycle of peptide screening, 270 peptides selected in accordance with the rule were synthesized and assayed. From the cell-based assay results, the highest scoring 20 peptides and the 20 lowest scoring

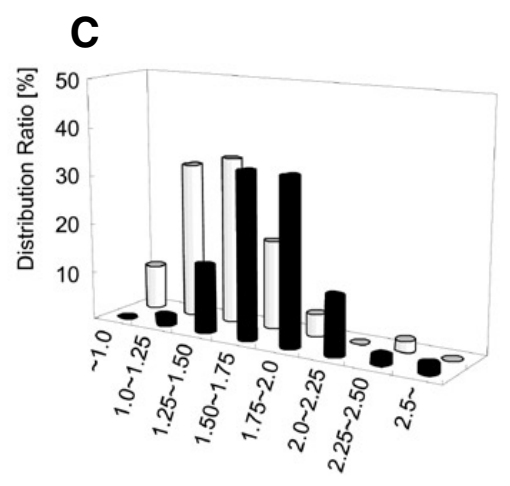

Range of Relative Cell-adhesion Ratio [-]

peptides were chosen as the "second original datasets" (Supplementary Table 2). The parameter selection and the model construction were carried out with the same condition as the first condition in the first cycle. From the best predictive model obtained from the second original dataset, a second rule for obtaining stronger celladhesion peptides from the peptides in accordance with the first rule was established. Fifty selected peptides were newly synthesized in accordance with the second rule and assayed.

In these processes, each newly synthesized peptide library on the peptide array functioned as a completely blind sequence outside the experimental data and provided new information for model adjustment during the screening.

\section{RESULTS}

\section{FNN-assisted Screening of Cell- adhesion Peptides}

A schematic diagram of FNNassisted peptide screening is presented in Figure 1. Our proposed strategy is the repeated cycling of two processes. The first process is the high-throughput peptide assay, which collects data as it
Figure 3. Evaluation of the peptide design rules for cell-adhesion. (A) Average value of relative cell-adhesion ratio with peptides synthesized in accordance with the deduced peptide design rules. The average value of the relative cell-adhesion ratio increased with peptide screening cycles but decreased with the reversed sequences. $* P<0.01, * * P<0.05$ (Utest). (B) Evaluation of the screening yield of high cell-adhesion ratio by repeated peptide screening cycles. The distributions of peptides newly synthesized in accordance with Rule 1 (black bar) and Rule 2 (grey bar) were compared with that of random sequences (no rule; white bar). (C) Evaluation of the deduced Rule 2 in the second peptide screening cycle. The distributions of 50 peptides synthesized in accordance with Rule 2 (black bar) and their reverse-sequence peptides (white bar) were compared. The relative cell-adhesion ratio was calculated as the ratio of the number of viable cells adhered on the peptide disk to no peptide blank disk. explores sequence space. The second process is computational modeling for obtaining indicative rules that guide the design of the following screen to achieve a higher yield. The first cycle was started from the first screening dataset, including the high-throughput data collection (Figure 1A). The screening data can be of limited size. For this study, we used a first screening dataset of only 643 peptides, which hardly covers the possible variation in 5-mer peptides, to confirm the efficiency of our strategy. From the first screening dataset of peptides, the top- and worst-ranked peptides were selected and converted into a numerical dataset (15 input parameters and the teacher signal) through the data conversion process (Figure 1B). Thus, peptide structural information was converted to input parameters. Since every residue is converted to physical properties (hydrophobicity, isoelectric point, and size) from exact sequence, the fuzziness of peptide structure is included in the model's construction. At the end of the first cycle, FNN assists the second screening process by extracting rules-the characteristic properties that differentiate positive sequences from negative sequences (Figure 1C). In this case, FNN provides the indicative rule for finding cell-adhesion peptides through 


\section{Research Reports}

learning the sequence tendencies in the screening data. Although based on limited screening data, this kind of FNN-derived rule enhances screening efficiency by reducing library size through restricting amino acid usage and increasing the yield from subsequent screening. In this study, the above-mentioned cycle was repeated twice, starting from the screening data obtained at the start of the cycle. We examined the screening yield in each cycle to evaluate the efficiency of the FNN-assisted screening.

From the first screening dataset, FNN model construction provided the best predictive model with the input parameter combination for obtaining cell-adhesion peptides: an amino acid residue of smaller size at $\mathrm{P} 1$, higher isoelectric point at $\mathrm{P} 2$, and smaller size at $\mathrm{P} 4$. The parameter combination is visualized as the rule matrix in Figure 2A (gray-colored cell) as Rule 1. Rule 1 was considered to be the most effective for cell-adhesion peptides, since the neighboring cell in the rule matrix was completely reversed, that is, it favored inactive peptides. By interpreting this rule, several restrictions for the following screening were deduced and the permitted amino acids at each important position were used (Table 1).

In accordance with Rule 1, the second cycle of screening started with the synthesis and assay of 270 tetramer peptides (the "second screening dataset"). Tetramer peptides were used because the design rule specified arrangements of four amino acids. The 270 peptides consisted of selected random sequences that were restricted in residue usage at chosen positions (P1, P2, and P4). The amino acid at the remaining position (P3) was randomly chosen from all 20 amino acids. As a result, the average relative celladhesion ratio among the 270 peptides following Rule 1 was 1.34 , and the yield of peptides scoring $\geq 1.5$-fold better than the controls was $31.5 \%$. As a comparison, the average relative cell-adhesion ratio among randomly selected 270 random peptides was 1.24 and the yield of peptides scoring $\geq 1.5$-fold better than the controls was $19.6 \%$ (Figure 3, A and B). This result strongly suggests that the rule derived by the FNN enhanced the yield of the screen using limited data.

From the assay results of the second screening dataset, the FNN extracted a new, adjusted rule: the peptide should have an amino acid of larger size and higher isoelectric point at P3 and one of higher isoelectric point at P1 (Rule 2; black-colored cell, Figure 2B). We used Rule 2 to guide the selection of amino acids at each important position in the peptides used in the next round of screening (see Table 1).

In accordance with Rule 2, we designed 50 new peptides (the third screening dataset), with limited amino acid usage at P1 and P3, and assayed their activity. The remaining positions (P2 and P4) were filled randomly from the set of amino acids indicated by Rule 1 . As a result, the average relative cell-adhesion ratio among 50 peptides designed by use of Rule 2 was 1.75 , and the yield of peptides scoring $\geq 1.5$-fold better than the controls was $84 \%$ (Figure 3, A and B). This result is a strong indication of the efficiency of our strategy for enhancing the screening yield, and demonstrates the importance of repeating the screening cycle rather than relying on the first predictive model constructed. This model study imitates actual screening experiments. We deduced that the first predictive model has limited predictive utility when there are only limited data relative to whole-coverage variation. Our strategy of using such predictions as indicators for the next screening may therefore work efficiently in actual screening.

\section{Evaluation of Indicative Rules Obtained from the FNN}

To further examine the usefulness of the obtained indicative rules established from FNN modeling, we synthesized a peptide array with the reversed sequences (e.g., the reversed sequence of RGDS is SDGR) of the 50 peptides synthesized in accordance with Rule 2 ("reversed data"). As a result, the relative adhesion ratio of the reversed sequence was low compared with that of the original sequence for most peptides. The average relative cell-adhesion ratio of the reversed sequences decreased 1.42 and the yield of peptides scoring $\geq 1.5$-fold better than the controls was $34 \%$. In addition, the corrected values (excluding the GRKV, VKRM, PRKM, GRRI, and VKKV peptides, which had reversed sequences that also conformed to Rule 2) were significantly lower, at 1.33 and $22.7 \%$ (Figure 3, $\mathrm{A}$ and C). This result indicates that peptide cell-adhesion activity was not related to total hydrophobicity or the peptide isoelectric point, and that the properties of the amino acids at each position are important. It also provides evidence that our strategy of screening using the FNN rules is effective.

\section{Novel Cell-adhesion Peptides Discovered in the Screening}

A list of newly identified peptides with the highest cell-adhesion activities is shown in Table 2. It should be noted that eight novel tetramer peptides (GKFQ, IRRC VKKP, PRKQ, GKKA, PKHQ, GKMN, and VKKV) that show relative cell-adhesion ratios higher than that of RGDS were found. This yield efficiency was strongly enhanced by our strategy of using FNN, the rule extractor, to guide peptide synthesis for subsequent screening.

\section{DISCUSSION}

We have proposed a strategy of using computational modeling for efficient screening with less experimental data. By focusing on peptide-cell interaction, which is rather weak and flexible, we have confirmed the efficiency of our strategy by increasing the yield of cell-adhesion peptides through two repeated screenings. We succeeded in increasing the yield of objective peptides up to $84 \%$, and obtained eight novel tetramer peptides that display higher cell-adhesion activities than the well-known cell-adhesion peptide RGDS from only $963(=643+270+50)$ screened peptides.

The most characteristic features of our method are the use of FNN, the computational prediction model, as a guide in subsequent screening, and the improvement of the model's predictive strength by the repetition and refining of experiments. Compared with other computational techniques developed 
to support peptide screening, our FNN algorithm has shown superior predictive accuracy when applied to various biological data $(9,14,33)$. Therefore, we believe that FNN is reliable compared with other algorithms. The advantage of our strategy using FNN is that it provides "rules" derived from the model structure. Drobni et al., also using predictive models to guide subsequent experiments, have demonstrated the effectiveness of a statistical molecular design approach in combination with quantitative structure activity relationship (QSAR) analyses in silico for the screening and design of new adhesion-inhibitory peptides of oral commensal bacteria (34). Through the QSAR modeling gained by partial least-squares (PLS) projection, amino acid properties that result in a change in biological response were deduced and can be used to inform the design of subsequent experiments. However, since the models were constructed using PLS analysis at each position, the combinatory effects of amino acids were cryptic and could not be predicted. Also, since the relationships between the properties of amino acids and the reactions were forcibly quantified, it is necessary to clarify the reliability of constructed models. Further, in spite of the capability of such structural modeling approaches to improve experimental design, the reliability of the modeling is critically dependent on the initial parameters, such as the solvent index. Therefore, for the model to enhance the actual yield of a screen, the experiments should be combined with appropriate cycles of model adjustment.

We obtained eight novel cell-adhesion peptides in our screening. We previously found some stronger celladhesion peptides from fibronectin-derived sequence, including the cell-adhesion motif RGDS. However we did not find peptides with activity exceeding RGDS, nor stronger alternative sequence derived from RGDS (15). Many researchers have reported tetramer or pentamer peptides that behave as cell-adhesion peptides. When a hexamer peptide library from fibronectin was used to screen for cell-adhesion peptides previously, a hexamer with an embedded RGDS was selected as one of the maximally active peptides. Hence, a tetramer or pentamer peptide is long enough to display good adhesion. It is known that RGDS binds to the $\alpha 5 \beta 1$ integrin subunit pair (35) among more than 24 known integrin subunit pairs. The peptides obtained in this study likely interact with a variety of cell surface receptors such as integrins. The exploration of novel motifs that may appear in other extracellular matrixes should contribute to the design of scaffolds for cell culture by allowing researchers to imitate the combinations of matrix components that occur in actual tissue.

By using only computational models constructed with huge amounts of data when attempting to enhance peptide screening, one risks the contradiction of requiring a huge amount of data beforehand. In this study, we used computational modeling based on small datasets effectively in combination with experiments. With the growing interest in screening and investigating orphan receptors, screening strategies that require huge amounts of data or confirmed seed sequences are not realistic. In such cases, we believe

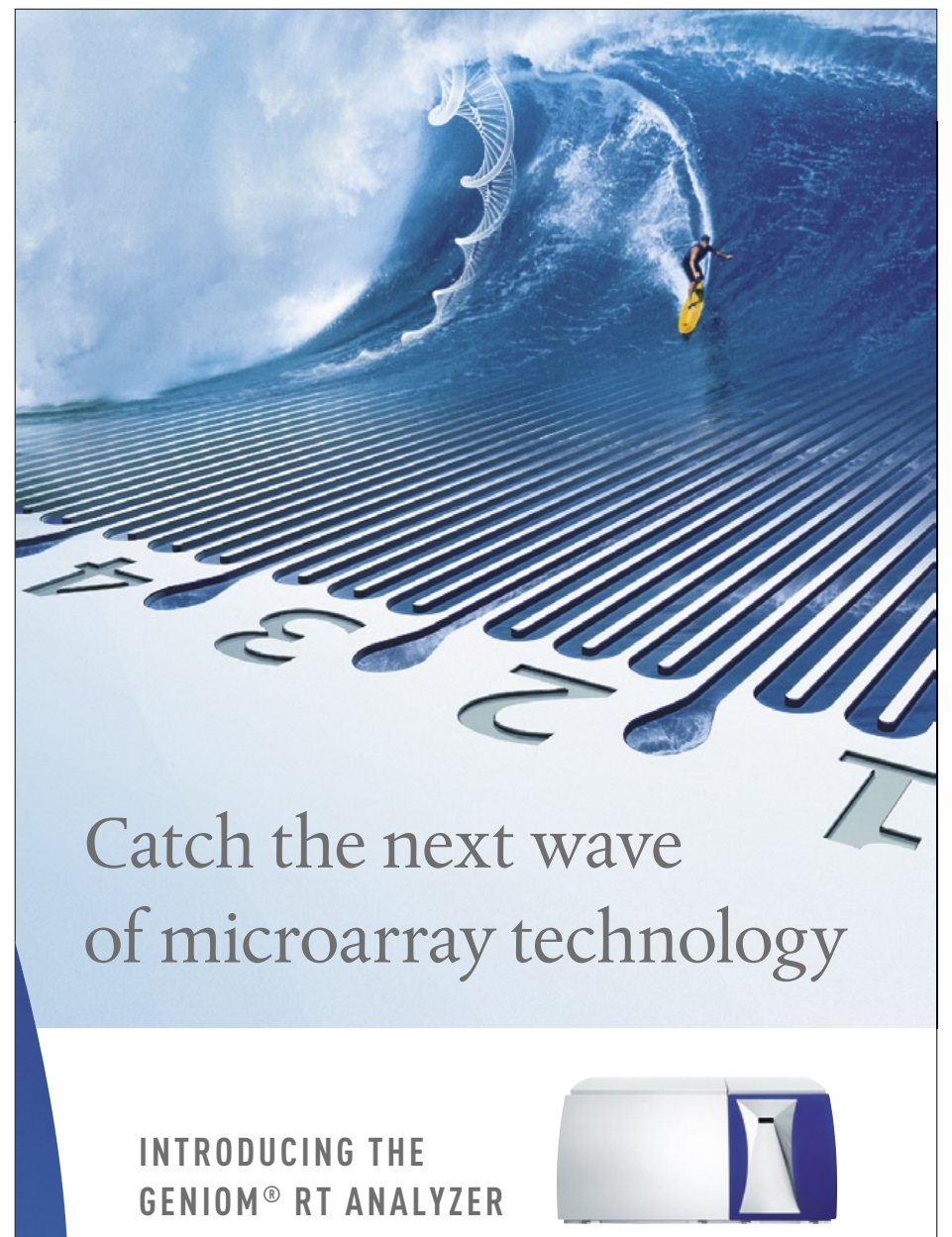

\section{AUTOMATED DYNAMIC MICROFLUIDICS}

Fully automated instrument for hybridization, washing, staining, and scanning of microarrays

High sensitivity through controlled active motion hybridization

Simple microarray handling

DESIGN YOUR ARRAY OR SELECT FROM OUR GROWING PORTFOLIO OF ARRAYS FOR

microRNA profiling

(include up-to-date versions of Sanger miRBase)

Expression profiling

Sequence capture for next generation sequencing
Learn about the

Geniom ${ }^{\circledR}$ RT Analyzer and

our comprehensive

services at analytica 2008 analytica2008 visit us: hall $A 3$, booth 410

\section{febit}

Europe: phone +496221 6510-300 - www.febit.eu - info@febit.eu Americas: phone +1 7813914360 -www.febit.com - info@febit.com 


\section{Research Reports}

that our strategy using FNN may greatly enhance screening.

\section{ACKNOWLEDGEMENTS}

This study was partly supported by a Grant-in-Aid for Scientific Research from the Japan Society for the Promotion of Science (JSPS; grant no. 17206082), and by a Research Fellowship at the JSPS to Chiaki Kaga.

\section{COMPETING INTERESTS STATEMENT}

The authors declare no competing interests.

\section{REFERENCES}

1. Burden, F.R. and D.A. Winkler. 2005. Predictive Bayesian neural network models of MHC class II peptide binding. J. Mol. Graph. Model. 23:481-489.

2. Brusic, V., G. Rudy, M. Honeyman, J. Hammer, and L. Harrison. 1998. Prediction of MHC class II-binding peptides using an evolutionary algorithm and artificial neural network. Bioinformatics 14:121-130.

3. Singh, H. and G.P. Raghava. 2001. ProPred: prediction of HLA-DR binding sites. Bioinformatics 17:1236-1237.

4. Tung, C.W. and S.Y. Ho. 2007. POPI: predicting immunogenicity of MHC class I binding peptides by mining informative physicochemical properties. Bioinformatics 23:942-949.

5. Salomon, J. and D.R. Flower. 2006. Predicting class II MHC-peptide binding: a kernel based approach using similarity scores. BMC Bioinformatics 7:501.

6. Zhang, G.L., I. Bozic, C.K. Kwoh, J.T. August, and V. Brusic. 2007. Prediction of supertype-specific HLA class I binding peptides using support vector machines. J. Immunol. Methods 320:143-154.

7. Ho, B.K. and K.A. Dill. 2006. Folding very short peptides using molecular dynamics. PLoS Comput Biol 2:e27.

8. Wu, J. and R.E. Aluko. 2007. Quantitative structure-activity relationship study of bitter di- and tri-peptides including relationship with angiotensin I-converting enzyme inhibitory activity. J. Pept. Sci. 13:63-69.

9. Noguchi, H., T. Hanai, H. Honda, L.C. Harrison, and T. Kobayashi. 2001. Fuzzy neural network-based prediction of the motif for MHC class II binding peptides. J. Biosci. Bioeng. 92:227-231.

10. Noguchi, H., R. Kato, T. Hanai, Y. Matsubara, H. Honda, V. Brusic, and T. Kobayashi. 2002. Hidden Markov modelbased prediction of antigenic peptides that interact with MHC class II molecules. J. Biosci. Bioeng. 94:264-270.
11. Horikawa, S., T. Furuhashi, Y. Uchiyama, and T. Tagawa. 1991. A study on fuzzy modeling using fuzzy neural networks. Proc. Int. Fuzzy Eng. Symp. 91:562-573.

12. Hanai, T. and H. Honda. 2004. Application of knowledge information processing methods to biochemical engineering, biomedical and bioinformatics fields. Adv. Biochem. Eng. Biotechnol. 91:51-73.

13. Kato, R., H. Nakano, H. Konishi, K. Kato, Y. Koga, T. Yamane, T. Kobayashi, and H. Honda. 2005. Novel strategy for protein exploration: high-throughput screening assisted with fuzzy neural network. J. Mol. Biol. 351:683-692.

14. Ando, T., M. Suguro, T. Kobayashi, M. Seto, and H. Honda. 2003. Multiple fuzzy neural network system for outcome prediction and classification of 220 lymphoma patients on the basis of molecular profiling. Cancer Sci. 94:906-913.

15. Kato, R., C. Kaga, M. Kunimatsu, T. Kobayashi, and H. Honda. 2006. Peptide array-based interaction assay of solid-bound peptides and anchorage-dependant cells and its effectiveness in cell-adhesive peptide design. J. Biosci. Bioeng. 101:485-495.

16. Kato, R., Y. Okuno, C. Kaga, M. Kunimatsu, T. Kobayashi, and H. Honda. 2005. Pentamer peptide from Fas antigen ligand inhibits tumor-growth with solid-bound form found by peptide array. J. Pept. Res. 66:146-153.

17. Okochi, M., M. Nakanishi, R. Kato, T. Kobayashi, and H. Honda. 2006. Highthroughput screening of cell death inducible short peptides from TNF-related apoptosis-inducing ligand sequence. FEBS Lett. 580:885-889

18. Frank, R. 2002. The SPOT-synthesis technique. Synthetic peptide arrays on membrane supports-principles and applications. J. Immunol. Methods 267:13-26.

19. Kramer, A. and J. Schneider-Mergener. 1998. Synthesis and screening of peptide libraries on continuous cellulose membrane supports. Methods Mol. Biol. 87:25-39.

20. Reineke, U., R. Volkmer-Engert, and J. Schneider-Mergener. 2001. Applications of peptide arrays prepared by the SPOT-technology. Curr. Opin. Biotechnol. 12:59-64.

21. Pierschbacher, M.D. and E. Ruoslahti. 1984. Cell attachment activity of fibronectin can be duplicated by small synthetic fragments of the molecule. Nature 309:30-33.

22. Graf, J., R.C. Ogle, F.A. Robey, M. Sasaki, G.R. Martin, Y. Yamada, and H.K. Kleinman. 1987. A pentapeptide from the laminin B1 chain mediates cell-adhesion and binds the 67,000 laminin receptor. Biochemistry 26:6896-6900.

23. Arap, W., R. Pasqualini, and E. Ruoslahti. 1998. Cancer treatment by targeted drug delivery to tumor vasculature in a mouse model. Science 279:377-380.

24. Kennel, S.J., S. Mirzadeh, G.B. Hurst, L.J. Foote, T.K. Lankford, K.A. Glowienka, L.L. Chappell, J.R. Kelso, et al. 2000. Labeling and distribution of linear peptides identified using in vivo phage display selection for tumors. Nucl. Med. Biol. 27:815825 .
25. Kelly, K.A., J.R. Allport, A. Tsourkas, V.R. Shinde-Patil, L. Josephson, and R. Weissleder. 2005. Detection of vascular adhesion molecule-1 expression using a novel multimodal nanoparticle. Circ. Res. 96:327336.

26. Frick, C., A. Odermatt, K. Zen, K.J. Mandell, H. Edens, R. Portmann, L. Mazzucchelli, D.L. Jaye, et al. 2005. Interaction of ICAM-1 with beta 2-integrin CD11c/CD18: characterization of a peptide ligand that mimics a putative binding site on domain D4 of ICAM-1. Eur. J. Immunol. 35:3610-3621.

27. Langer, R. and D.A. Tirrell. 2004 Designing materials for biology and medicine. Nature 428:487-492.

28. Lutolf, M.P. and J.A. Hubbell. 2005 Synthetic biomaterials as instructive extracellular microenvironments for morphogenesis in tissue engineering. Nat. Biotechnol. 23:4755 .

29. Khademhosseini, A., R. Langer, J. Borenstein, and J.P. Vacanti. 2006 Microscale technologies for tissue engineering and biology. Proc. Natl. Acad. Sci. USA 103:2480-2487.

30. Kyte, J. and R.F. Doolittle. 1982. A simple method for displaying the hydropathic character of a protein. J. Mol. Biol. 157:105-132.

31. Fauchere, J.L., M. Charton, L.B. Kier, A. Verloop, and V. Pliska. 1988. Amino acid side chain parameters for correlation studies in biology and pharmacology. Int. J. Pept. Protein Res. 32:269-278.

32. Zimmerman, J.M., N. Eliezer, and R. Simha. 1968. The characterization of amino acid sequences in proteins by statistical methods. J. Theor. Biol. 21:170-201.

33. Takahashi, H. and H. Honda. 2006 Prediction of peptide binding to major histocompatibility complex class II molecules through use of boosted fuzzy classifier with SWEEP operator method. J. Biosci. Bioeng. 101:137-141.

34. Drobni, M., I.M. Olsson, C. Eriksson, F. Almqvist, and N. Stromberg. 2006. Multivariate design and evaluation of a set of RGRPQ-derived innate immunity peptides. J. Biol. Chem. 281:15164-15171.

35. Hynes, R.O. 2002. Integrins: bidirectional, allosteric signaling machines. Cell 110:673687.

Received 16 May 2007; accepted 5 November 2007.

Address correspondence to Hiroyuki Honda, Department of Biotechnology, School of Engineering, Nagoya University, Furocho, Chikusa-ku, Nagoya 464-8603, Japan. e-mail: honda@nubio.nagoya-u.ac.jp

To purchase reprints of this article, contact: Reprints@BioTechniques.com 